\title{
On Solutions of a Higher Order Nonhomogeneous Ordinary Differential Equation
}

\author{
Elif Nuray Yıldırım $^{1 *}$ and Ali Akgül ${ }^{2}$ \\ ${ }^{1}$ Department of Mathematics, Faculty of Humanities and Social Sciences, Istanbul Commerce University, Istanbul, Turkey \\ ${ }^{2}$ Department of Mathematics, Faculty of Science and Arts, Siirt University, Siirt, Turkey \\ * Corresponding author
}

\begin{abstract}
Article Info
Keywords: Approximate solution, Initial value problems Reproducing kernel method, Nonhomogeneous ordinary differential equations

2010 AMS: 47B32, 46E22, 35M32,

74530

Received: 15 September 2020

Accepted: 24 November 2020

Available online: 15 December 2020
\end{abstract}

Abstract

\begin{abstract}
Higher order differential equations (ODE) has an important role in the modelling process. It is also much significant which the method is used for the solution. In this study, in order to get the approximate solution of a nonhomogeneous initial value problem, reproducing kernel Hilbert space method is used. Reproducing kernel functions have been obtained and the given problem transformed to the homogeneous form. The results have been presented with the graphics. Absolute errors and relative errors have been given in the tables.
\end{abstract}

\section{Introduction}

In this study, by using reproducing kernel method we aim to find the approximate solution of the problem in the form as:

$$
(\mathbb{L} h)(x)=p(x) h^{\prime \prime \prime}(x)+q(x) h^{\prime}(x)=f(x), a \leq x \leq b
$$

The reproducing kernel method (RKM) have been used as an efficient way to solve different types of differential equations by many researcher for years. The theory of RKM was begin with the research of Aronszajn and Bergman [1, 2]. Since the method is very effective, many researcher applied the method to the several kind of problems. For instance Cui et al. [3] published a book about numerical analysis in the reproducing kernel space which is a comprehensive study. Syam et al. [4] used the method to solve a class of fractional Sturm-Liouville eigenvalue problems.

Jiang and Tian [5] solved the Volterra integro-differential equations of fractional order by the reproducing kernel method. Li et al. [6] applied the method for numerical solutions of fractional Riccati differential equations. For more details see [7]-[9].

In many models and problems, the equations need to be solved numerically. Therefore many approaches have been used and there have been lots of efforts for solving non-linear higher order ordinary differential equations in researches. For instance, Homotopy perturbation method [10], Adomian decomposition method [11], Chebyshev collocation method [12] used. Adomian decomposition method for solving initial value problems in second-order ordinary differential equations is given in [13]. Lu et al. Furthermore Runge- Kutta method [14], Predictor-Corrector method [15], decomposition method [16], direct block method [17], have been used for solving IVP. For a further reading and more details one can see [18]-[26]. 


\section{RKM for higher order nonhomogeneous ordinary differential equations}

In this section, we will discuss the solution of a class of higher order ODEs for IVPs in the following form:

$$
\begin{gathered}
(\mathbb{L} h)(x)=p(x) h^{\prime \prime \prime}(x)+q(x) h^{\prime}(x)=f(x), a \leq x \leq b \\
h^{i}(a)=\gamma_{i}, 0 \leq i \leq 2
\end{gathered}
$$

where $p(x)$ and $q(x)$ are continuous functions on the interval $[a, b]$. With the purpose of finding the solution of the problem (2.1) by using RKM (reproducing kernel method), we first need to define the RKS(reproducing kernel space) to which the equation belongs. The space construction is relevant to the order and conditions of the ODE that wanted to solved.

\section{Construction of the method}

To be able to solve the problem (2.1) using proposed method which we will denote RKM, we first construct reproducing kernel spaces. After the space construction, we will close to the approximate solution by obtaining the reproducing kernel functions belong to the given differential equation. In this section, we present some essential definitions and theorems in the theory of suggested method.

Definition 3.1 (Reproducing Kernel). [2] Let $Q$ be a nonempty set. A function $R: Q \times Q \rightarrow \mathbb{F}$ is called a reproducing kernel of the Hilbert space $\mathscr{H}$ if and only if

(a) $R(\cdot, x) \in \mathscr{H}, \quad \forall x \in Q$,

(b) $\langle\gamma, R(\cdot, x)\rangle=\gamma(x)$.

The item $(b)$ is called "reproducing property" of kernel $R$. The value of the function $\gamma$ at the point $x$ is reproduced by the inner product of $\gamma$ with $R(\cdot, x)$.

Definition 3.2. [3] The space $S_{2}^{m}[a, b]$ consist of the functions $h:[a, b] \rightarrow \mathbb{R}$ and define as follows:

$$
S_{2}^{m}[a, b]=\left\{h(x) \mid h^{(m-1)}(x) \in A C[a, b], \quad h^{(m)}(x) \in L^{2}[a, b], \quad x \in[a, b]\right\} .
$$

$S_{2}^{m}[a, b]$ equipped with the inner product

$$
<h, t>_{S_{2}^{m}}=\sum_{i=0}^{m-1} h^{(i)}(a) t^{(i)}(a)+\int_{a}^{b} h^{(m)}(x) t^{(m)}(x) d x .
$$

Here we denote the vector space of absolutely continuous (real-valued) functions with $A C[a, b]$ and the quadratically integrable functions on the interval $[a, b]$ with $L^{2}[a, b]$.

Lemma 3.3. If a Hilbert space has a reproducing kernel, it is called a reproducing kernel Hilbert space (RKHS).

Lemma 3.4. $[3] S_{2}^{m}[a, b]$ function space is a reproducing kernel space.

The reproducing kernel function of the space $S_{2}^{m}$ can be written as:

$$
R_{x}(y)=\left\{\begin{array}{l}
R(x, y)=\sum_{i=1}^{2 m} h_{i}(y) x^{i-1}, x \leq y, \\
R(y, x)=\sum_{i=1}^{2 m} t_{i}(y) x^{i-1}, x>y .
\end{array}\right.
$$

For the proof of Lemma 3.4 one can see [3].

In the next subsection, we present a special reproducing kernel function space on the interval $[0,4 \pi]$. 


\section{1. $S_{2}^{4}[0,4 \pi]$ Reproducing kernel space}

According to the reproducing kernel theory, we construct the space with concerning of the order of the derivative in the problem. For this reason in the equation (3.1) we choose $m=4$. Let we now define the function space

$$
S_{2}^{4}[0,4 \pi]=\left\{h(x) \mid h^{\prime \prime}(x) \in A C[0,4 \pi], \quad h^{\prime \prime \prime}(x) \in L^{2}[0,4 \pi], \quad x \in[0,4 \pi]\right\}
$$

with the inner product

$$
\begin{aligned}
<h, R_{y}>_{S_{2}^{4}[0,4 \pi]} & =h(0) R_{y}(0)+h^{\prime}(0) R_{y}^{\prime}(0)+h^{\prime \prime}(0) R_{y}^{\prime \prime}(0)-h^{(3)}(0) R_{y}^{(3)}(0) \\
& +\int_{0}^{4 \pi}\left(h(x)+(4)(x) R_{y}^{(4)}(x)\right) d x .
\end{aligned}
$$

Integrating this equation by parts for four times, we have

$$
\begin{aligned}
<h, R_{y}>_{S_{2}^{4}[0,4 \pi]} & =h(0) R_{y}(0)+h^{\prime}(0) R_{y}^{\prime}(0)+h^{\prime \prime}(0) R_{y}^{\prime \prime}(0)-h^{(3)}(0) R_{y}^{(3)}(0) \\
& +h^{(3)}(4 \pi) R_{y}^{(4)}(4 \pi)-h^{(3)}(0) R_{y}^{(4)}(0)-h^{\prime \prime}(4 \pi) R_{y}^{(5)}(4 \pi) \\
& +h^{\prime \prime}(0) R_{y}^{(5)}(0)+h^{\prime}(4 \pi) R_{y}^{(6)}(4 \pi)-h^{\prime}(0) R_{y}^{(6)}(0) \\
& -h(4 \pi) R_{y}^{(7)}(4 \pi)+h(0) R_{y}^{(7)}(0)+\int_{0}^{4 \pi} h(x) R_{y}^{(8)}(x) d x .
\end{aligned}
$$

Because of the conditions, we get the following equations:

$$
\begin{aligned}
& \text { 1. } R_{y}(0)=0 \\
& \text { 2. } R_{y}^{\prime}(0)=0 \\
& \text { 3. } R_{y}^{\prime \prime}(0)=0 .
\end{aligned}
$$

With these three functions being zero we obtain:

$$
\begin{aligned}
<h, R_{y}>_{S_{2}^{4}[0,4 \pi]} & =h^{(3)}(0) R_{y}^{(3)}(0)+h^{(3)}(4 \pi) R_{y}^{(4)}(4 \pi)-h^{(3)}(0) R_{y}^{(4)}(0) \\
& -h^{\prime \prime}(4 \pi) R_{y}^{(5)}(4 \pi)+h^{\prime}(4 \pi) R_{y}^{(6)}(4 \pi)-h(4 \pi) R_{y}^{(7)}(4 \pi) \\
& +\int_{0}^{4 \pi} h(x) R_{y}^{(8)}(x) d x .
\end{aligned}
$$

When the equation is rearranged we get the following equations:
4. $R_{y}^{(3)}(0)-R_{y}^{(4)}(0)=0$
5. $R_{y}^{(4)}(4 \pi)=0$
6. $R_{y}^{(5)}(4 \pi)=0$
7. $R_{y}^{(6)}(4 \pi)=0$
8. $R_{y}^{(7)}(4 \pi)=0$.

Then we will get:

$$
<h, R_{y}>_{S_{2}^{4}[0,4 \pi]}=\int_{0}^{4 \pi} h(x) R_{y}^{(8)}(x) d x
$$

With the knowledge of reproducing kernel property, the function $u(y)$ can be written in the form:

$$
<h, R_{y}>_{S_{2}^{4}[0,4 \pi]}=h(y) .
$$


For this reason, we reach

$$
\int_{0}^{4 \pi} h(x) R_{y}^{(8)}(x) d x=h(y)
$$

Because of the definition of Dirac-Delta function, it is obvious that the equation (3.2) is equal to the $\delta(x-y)$. That gives us the following equation:

$$
R_{y}^{(8)}(x)=\delta(x-y)
$$

When $x \neq y$, the reproducing kernel function $R_{y}$ can be written in the form as:

$$
R_{y}(x)=\left\{\begin{array}{l}
\sum_{k=1}^{8} c_{k} x^{k-1}, x \leq y \\
\sum_{k=1}^{8} d_{k} x^{k-1}, x>y
\end{array}\right.
$$

By using the feature of Dirac-Delta function, the following equations can be written:

$$
\begin{aligned}
& \text { 9. } R_{y^{+}}(y)=R_{y^{-}}(y) \\
& \text { 10. } R_{y^{+}}^{\prime}(y)=R_{y^{-}}^{\prime}(y) \\
& \text { 11. } R_{y^{+}}^{\prime \prime}(y)=R_{y^{-}}^{\prime \prime}(y) \\
& \text { 12. } R_{y^{+}}^{\prime \prime \prime}(y)=R_{y^{-}}^{\prime \prime \prime}(y) \\
& \text { 13. } R_{y^{+}}^{(4)}(y)=R_{y^{-}}^{(4)}(y) \\
& \text { 14. } R_{y^{+}}^{(5)}(y)=R_{y^{-}}^{(5)}(y) \\
& \text { 15. } R_{y^{+}}^{(6)}(y)=R_{y^{-}}^{(6)}(y) \\
& \text { 16. } R_{y^{+}}^{(7)}(y)-R_{y^{-}}^{(7)}(y)=1 .
\end{aligned}
$$

In order to find the reproducing kernel function of the given space, we need to solve the differential equation system above. For this purpose, we needed sixteen equation since the (3.1) has sixteen coefficients and we obtained them.If we solve the system thus we get the reproducing kernel function as:

$$
R_{y}(x)=\frac{1}{36} y^{3} x^{3}+\frac{1}{144} y^{3} x^{4}-\frac{1}{240} y^{2} x^{5}+\frac{1}{720} y x^{6}-\frac{1}{5040} x^{7}
$$

The proof of the following theorem is similar to the proof of the Lemma 3.4, so we omit it.

Theorem 3.5. The function space $S_{2}^{4}[0,4 \pi]$ is a reproducing kernel Hilbert space.

Reproducing kernel function and the RKS has a vital role in the way to the solution. In the next section we give other essential part of the method.

\section{Implementation of the method}

Firstly, we define the linear operator as

$$
\mathbb{L}: S_{2}^{m}[0,4 \pi] \longrightarrow S_{2}^{m-n}[0,4 \pi]
$$

such that

$$
\mathbb{L} h(x)=K\left(x, h(x), h^{\prime}(x), \ldots, h^{(n)}(x)\right) .
$$

It is known that the operator $\mathbb{L}$ is bounded.

After the operator, we now construct the orthogonal system of the space $S_{2}^{m}[0,4 \pi]$. Let $\eta_{i}(x)=\overline{R_{x_{i}}}(x)$ and $\psi_{i}(x)=\mathbb{L}^{*} \eta_{i}(x)$. Here, $\mathbb{L}^{*}$ is adjoint operator of $\mathbb{L}$ and the set of $x_{i}$ which denoted by $\left\{x_{i}\right\}_{j=1}^{\infty}$ is dens in the interval $[0,4 \pi]$. 
Theorem 4.1. If $\left\{x_{i}\right\}_{i=1}^{\infty}$ is dense in the $[0,4 \pi]$, then $\left\{\psi_{i}(x)\right\}_{i=1}^{\infty}$ is a complete system of $S_{2}^{m}[0,4 \pi]$.

Proof. Let $\left\{x_{i}\right\}_{j=1}^{\infty}$ is dense in the interval $[0,4 \pi]$. By using adjoint operator and reproducing kernel properties we can write

$$
\left\langle h(x), \psi_{i}(x)\right\rangle=\left\langle h(x), \mathbb{L}^{*} \eta_{i}(x)\right\rangle=\left\langle\mathbb{L} h(x), \eta_{i}(x)\right\rangle=h\left(x_{i}\right)=0, i=1,2, \ldots
$$

With the knowledge of density of $\left\{x_{i}\right\}_{j=1}^{\infty}$ and considering the continutiy of $h(x)$, we arrive that $h(x)=0$.

In order to obtain the approximate solution, with the help of Gram-Schimidt orthogonalization process, the orthonormal system $\left\{\psi_{i}(x)\right\}_{j=1}^{\infty}$ need to be construct. It can be denoted as:

$$
\left\{\bar{\psi}_{i}(x)\right\}_{j=1}^{\infty}=\sum_{r=1}^{i} \zeta_{i r} \psi_{r}(x), \zeta_{i i}>0,(i=1,2, \ldots)
$$

Here the function $\zeta_{i k}$ represents the orthogonal coefficients.

Theorem 4.2. Let $\left\{x_{i}\right\}_{j=1}^{\infty}$ be dense in $[0,4 \pi]$.If the problem (2.1) has a uniqe solution then it can be denoted as follow:

$$
h(x)=\sum_{i=1}^{\infty} \sum_{r=1}^{i} \zeta_{i r} K\left(x_{r}, h\left(x_{r}\right), h^{\prime}\left(x_{r}\right), \ldots, h^{(n)}\left(x_{r}\right)\right) \bar{\psi}_{i}(x) .
$$

Proof. Let we choose the solution of the equation (2.1) as $h(x)$. By knowing that $\left\{\bar{\psi}_{i}(x)\right\}_{i=1}^{\infty}$ is the orthonormal basis of the space, we can write the following equality:

$$
h(x)=\sum_{i=1}^{\infty}\left\langle h(x), \bar{\psi}_{i}(x)\right\rangle \bar{\psi}_{i}(x)=\sum_{i=1}^{\infty} \sum_{r=1}^{i} \zeta_{i r}\left\langle h(x), \bar{\psi}_{i}(x)\right\rangle \bar{\psi}_{i}(x) .
$$

Let we now do apply the feature of adjoint operator at this step.

$$
\begin{aligned}
\sum_{i=1}^{\infty} \sum_{r=1}^{i} \zeta_{i r}\left\langle h(x), \mathbb{L}^{*} \eta_{r}(x)\right\rangle \bar{\psi}_{i}(x) & =\sum_{i=1}^{\infty} \sum_{r=1}^{i} \zeta_{i r}\left\langle\mathbb{L} h(x), \eta_{r}(x)\right\rangle \bar{\psi}_{i}(x) \\
& =\sum_{i=1}^{\infty} \sum_{r=1}^{i} \zeta_{i r}\left\langle K\left(x, h(x,) h^{\prime}(x), \ldots, h^{(n)}, \eta_{r}(x)\right\rangle \bar{\psi}_{i}(x)\right. \\
& =\sum_{i=1}^{\infty} \sum_{r=1}^{i} \zeta_{i r} K\left(x_{r}, h\left(x_{r}\right), h^{\prime}\left(x_{r}\right), \ldots, h^{(n)}\left(x_{r}\right)\right) \bar{\psi}_{i}(x)
\end{aligned}
$$

With the last equation the proof is completed.

\section{Application and numerical results}

In this section we will apply the proposed method to the problem which in the form (2.1). By aiming to find the approximate solution we will use suitable reproducing kernel Hilbert space and kernel functions which belongs to the space. Once we obtained the solution we will present the absolute and relative errors in the tables. Let us begin by considering the initial value problem given below:

$$
\begin{gathered}
h^{\prime \prime \prime}(x)+4 h^{\prime}(x)=x, 0 \leq x \leq 4 \pi \\
h(0)=h^{\prime}(0)=0, h^{\prime \prime}(0)=1 .
\end{gathered}
$$

The exact solution of the equation is

$$
h(x)=\frac{3}{16}(1-\cos 2 x)+\frac{1}{8} x^{2}
$$

We seek the solution function $h$ of the form $h(x)=H(x)+S(x)$. This will ensure that the new boundary conditions are homogeneous. Here $S(x)$ denotes the transformation function which satisfies the initial conditions and $H(x)$ denotes the terms of new initial value problem which will be homogeneous. If we do the required arrangements, we will be obtaining the new homogeneous equation with the homogeneous conditions. 
As a first step, let we start to transform the conditions of the equation (5.1) to the homogeneous one. For this purpose let we use the transformation function given follow as:

$$
S(x)=\frac{x^{2}}{2}
$$

So we can write the $h$ function as:

$$
h(x)=H(x)+\frac{x^{2}}{2}
$$

If we calculate the necessary derivatives then we get

$$
\begin{aligned}
h^{\prime}(x) & =H^{\prime}(x)+x \\
h^{\prime \prime}(x) & =H^{\prime \prime}(x)+1 \\
h^{\prime \prime \prime}(x) & =H^{\prime \prime \prime}(x) .
\end{aligned}
$$

When we put this equations into the main problem (5.1), the equation will transform to the new version which is homogeneous as:

$$
H^{\prime \prime \prime}(x)+4 H^{\prime}(x)=-3 x, 0 \leq x \leq 4 \pi
$$

with the initial conditions

$$
H(0)=H^{\prime}(0)=H^{\prime \prime}(0)=0
$$

By using the reproducing kernel function which found in section 3.1 and with the help of the programme Matlab we obtained the approximate solutions of the problem (5.1). By taking the dense point $\mathrm{M}=100$, the exact solution and approximate solution compared. Besides, absolute and relative errors of the results are presented in the Table 1, Table 2 and graphics below.

\begin{tabular}{lll}
\hline $\mathbf{x}$ & Exact & RKM (App.) \\
\hline 0.1 & 0 & 0 \\
\hline 0.2 & 0.004987516700 & 0.004986813148 \\
\hline 0.3 & 0.01980106360 & 0.01979816350 \\
\hline 0.4 & 0.04399957220 & 0.04399305315 \\
\hline 0.5 & 0.07686749200 & 0.07685607747 \\
\hline 0.6 & 0.1174433176 & 0.1174259428 \\
\hline 0.7 & 0.1645579210 & 0.1645337905 \\
\hline 0.8 & 0.2168811607 & 0.2168497896 \\
\hline 0.9 & 0.2729749104 & 0.2729361522 \\
\hline 1.0 & 0.3313503928 & 0.3313044491 \\
\hline
\end{tabular}

Table 1: Exact solution and approximate solution. 


\begin{tabular}{lll}
\hline $\mathbf{x}$ & $\mathbf{R K M}(\mathbf{A E})$ & $\mathbf{R K M}(\mathbf{R E})$ \\
\hline 0.1 & 0 & 0 \\
\hline 0.2 & $7.03552 \cdot 10^{-7}$ & 0.0001410625853 \\
\hline 0.3 & 0.00000290010 & 0.0001464618295 \\
\hline 0.4 & 0.00000651905 & 0.0001481616678 \\
\hline 0.5 & 0.00001141453 & 0.0001484961939 \\
\hline 0.6 & 0.0000173748 & 0.0001479420060 \\
\hline 0.7 & 0.0000241305 & 0.0001466383378 \\
\hline 0.8 & 0.0000313711 & 0.0001446464963 \\
\hline 0.9 & 0.0000387582 & 0.0001419844774 \\
\hline 1.0 & 0.0000459437 & 0.0001386559394 \\
\hline
\end{tabular}

Table 2: Absolute Errors (AE) and Relative Errors (RE).

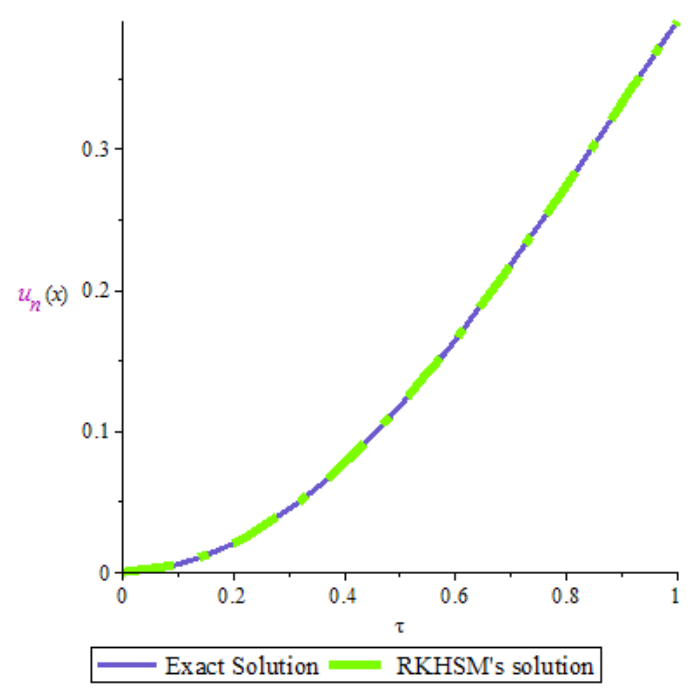

Figure 5.1: 2D Comparison between exact solution and RKM solution

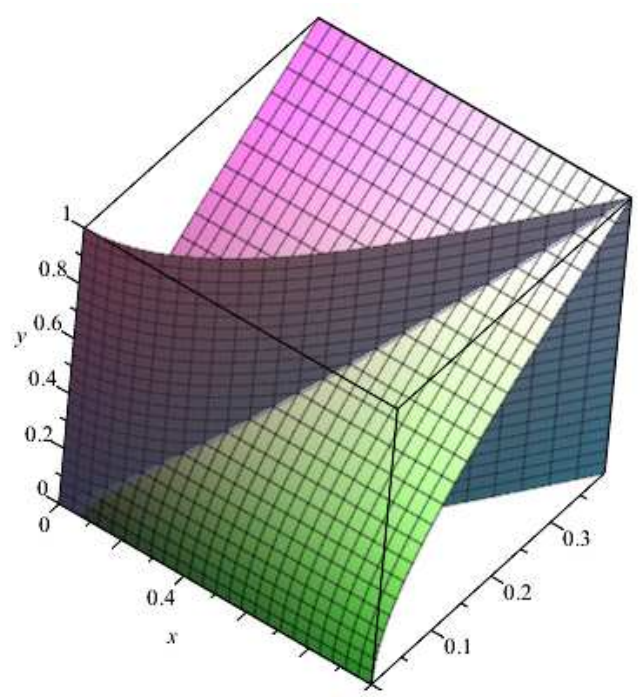

Figure 5.2: 3D Comparison 


\section{Conclusions}

In this work, we presented the reproducing kernel method for solving an IVP which is a type of higher order differential equations. We found a new reproducing kernel space and used it to obtain the numerical results of the problem. When the exact solution and the approximate solution compared, it can easily seen that the method works quite well. The absolute and relative errors also a proof of that. As a result, it is clear that the method is effective and smooth. Moreover, it is an undeniable advantage that the method gives the result fast. This prevents waste of time and provides fast access to the solution.

\section{References}

[1] N. Aronszajn, Theory of reproducing kernels, Trans. Amer. Math. Soc. 68 (1950), 337-404.

[2] S. Bergman, The Kernel Function and Conformal Mapping, American Math. Soc., New York, (1950).

[3] M. Cui, Y. Lin, Nonlinear Numerical Analysis in the Reproducing Kernel Space, New York: Nova Sci. Publ., (2009).

[4] M. I. Syam, Q. M. Al-Mdallal and M. Al-Refai, A numerical method for solving a class of fractional Sturm-Liouville eigenvalue problems, Commun. Numer. Anal., 2 (2017), 217-232

[5] W. Jiang, T. Tian, Numerical solution of nonlinear Volterra integro-differential equations of fractional order by the reproducing kernel method, App. Math. Mod., 39 (16) (2015), 4871-4876.

[6] X. Y. Li, B. Y. Wu, R. T. Wan, Reproducing kernel method for fractional Riccati differential equations, Abstr. Appl. Anal., (2014), 1-6.

[7] A. Alvandi, M. Paripour, The combined reproducing kernel method and Taylor series to solve nonlinear Abel's integral equations with weakly singular kernel, Cogent Mathematics, 3 (2016).

[8] A. Freihat, R. Abu-Gdairi, H. Khalil, E. Abuteen, M. Al-Smadi, R. A. Khan, Fitted reproducing kernel method for solving a class of third-order periodic boundary value problems, American J. App. Sci., 13 (2016), 501-510.

[9] G. Akram, H. U. Rehman, Numerical solution of eighth order boundary value problems in reproducing Kernel space, Numer. Algor, 62(3) (2013),

[10] S. Abbasbandy, Homotopy perturbation method for quadratic Riccati differential equation and comparison with Adomian's decomposition method, Appl. Math. Comput, 172 (2006), 485-490.

[11] G. Adomian, Nonlinear Stochastic Operator Equations, Academic Press, San Diego, (1986).

[12] A. Daşçığlu, H. Yaslan, The solution of high-order nonlinear ordinary differential equations by Chebshev series, Appl. Math. Comput., 217 (2011),

5658-5666.
[13] A.M. Wazwaz, A new method for solving initial value problems in second-order ordinary differential equations, Appl. Math. Comput., 128 (2002),

[14] M. K. Horn, Fourth- and fifth-order, scaled Runge-Kutta algorithms for treating dense output, SI AM J. Numer. Analysis, 20 (1983), $558-568$.

[15] L. Fox, D. F. Mayers, Numerical Solution of Ordinary Differential Equations, Chapman and Hall, (1987).

[16] A.M. Wazwaz, The numerical solution of fifth-order boundary value problems by the decomposition method, J. Comput. Appl. Math. 136(1-2) (2001),

[17] Waeleh et al., Numerical solution of higher order ordinary differential equations by direct block code, J. Math. Sta., 8(1) (2012), 77-81.

[18] E. A. Coddington, N. Levinson, Theory of Ordinary Differential Equations, Tata McGraw-Hill Publishing, (1972).

[19] F. Hoppensteadt, Properties of solutions of ordinary differential equations with small parameters, Commun. Pure Appl. Anal., 24(6) (1971), 807-840.

[20] G. R. Sell, On the fundamental theory of ordinary differential equations, J. Differential Equations, 1 (1965), $370-392$.

[21] D. Baleanu, A. Fernandez, A. Akgül, On a fractional operator combining proportional and classical differintegrals, Mathematics, 8(3) (2020).

[22] A. Akgül, A novel method for a fractional derivative with non-local and non-singular kernel, Chaos, Solitons Fractals 114, (2020), 478-482.

[23] E. K. Akgül, Solutions of the linear and nonlinear differential equations within the generalized fractional derivatives, Chaos: An Inter. J. Nonlin. Sci. 29(2) 023108, (2020).

[24] K. M. Owolabi, A. Atangana, A. Akgül, Modelling and analysis of fractal-fractional partial differential equations: Application to reaction-diffusion model, Alexandria Eng. J. 59 (2020), 2477-2490.

[25] A. Atangana, A. Akgül, K. M. Owolabi, Analysis of fractal fractional differential equations, Alexandria Eng. J. 59 (2020), $1117-1134$.

[26] A. Atangana, A. Akgül, Can transfer function and Bode diagram be obtained from Sumudu transform, Alexandria Eng. J. 59 (2020), $1971-1984$. 